\title{
RADAR Reference Adult, Pediatric, and Pregnant Female Phantom Series for Internal and External Dosimetry
}

\author{
Michael G. Stabin ${ }^{1}$, X. George Xu ${ }^{2}$, Mary A. Emmons ${ }^{1}$, W. Paul Segars ${ }^{3}$, Chengyu Shi $^{2}$, and Michael J. Fernald ${ }^{1}$ \\ ${ }^{1}$ Vanderbilt University, Nashville, Tennessee; ${ }^{2}$ Rensselaer Polytechnic Institute, Troy, New York; and ${ }^{3}$ Duke University, Durham, \\ North Carolina
}

A new generation of reference computational phantoms, based on image-based models tied to the reference masses defined by the International Commission on Radiological Protection (ICRP) for dose calculations, is presented. Methods: Anatomic models based on nonuniform rational b-spline modeling techniques were used to define reference male and female adults, 15-y-olds, 10-y-olds, 5-y-olds, 1-y-olds, newborns, and pregnant women at 3 stages of gestation, using the defined reference organ masses in ICRP publication 89. Absorbed fractions and specific absorbed fractions for internal emitters were derived using standard Monte Carlo radiation transport simulation codes. Results: Differences were notable between many pairs of organs in specific absorbed fractions because of the improved realism of the models, with adjacent organs usually closer and sometimes touching. Final estimates of absorbed dose for radiopharmaceuticals, for example, were only slightly different overall, as many of the differences were small and most pronounced at low radiation energies. Some new important organs were defined (salivary glands, prostate, eyes, and esophagus), and the identity of a few gastrointestinal tract organs changed. Conclusion: A new generation of reference models for standardized internal and external dose calculations has been defined. The models will be implemented in standardized software for internal dose calculations and be used to produce new standardized dose estimates for radiopharmaceuticals and other applications.

Key Words: computational phantoms; dosimetry; Monte Carlo calculations

J Nucl Med 2012; 53:1807-1813

DOI: 10.2967/jnumed.112.106138

A nthropomorphic models-also called computational phantoms - applied for dose calculations in nuclear medicine for the past $30 \mathrm{y}$ have used the stylized geometric definitions that were developed for reference adults and children $(1)$ and pregnant women (2). Figure 1 shows an exterior view of one

Received Mar. 20, 2012; revision accepted May 29, 2012

For correspondence or reprints contact: Michael G. Stabin, Department of Radiology and Radiological Sciences, Vanderbilt University, 116121 st Ave.

S., Nashville, TN 37232-2675.

E-mail: michael.g.stabin@vanderbilt.edu

Published online Sep. 11, 2012.

COPYRIGHT (C) 2012 by the Society of Nuclear Medicine and Molecular Imaging, Inc. of the Cristy/Eckerman stylized adult and pediatric models. The models' exteriors were defined in 3 sections: an elliptic cylinder representing the arm, torso, and hips; a truncated elliptic cone representing the legs and feet; and an elliptic cylinder representing the head and neck. Several organs and tissues were mathematically defined as occupying finite spaces within the body space and comprised 3 types of tissue: soft tissue, bone, and lung. The mathematic descriptions of the organs were formulated on the basis of descriptive and schematic materials from general anatomy references. This series of stylized models was implemented in the MIRDOSE (3) and OLINDA/EXM 1.0 (4) personal computer codes to facilitate calculation of standardized internal dose calculations.

The stylized models that originated at Oak Ridge National Laboratory some 40 y ago have now been replaced with realistic body models based mostly on human image data (5). The image-based models are now considered to be the second-generation anatomic models that use the so-called voxel geometry. Petoussi-Henss et al. created tomographic voxelized models from CT images (6) and found that the stylized computational models could over- and underestimate organ absorbed fractions by several tens of percentage points when compared against the more realistic, image-based models. Similar discrepancies between the stylized and voxelized models were reported using the VIP-Man model, which is based on the Visible Human Project cadaver cross-sectional anatomic images $(7,8)$. Hurtado et al. (9) reported on hybrid realistic computational phantoms for adults and used them in retrospective dosimetry analyses for contaminated radiation workers. Many others have developed individual realistic phantoms of adults $(10,11)$, children (12), and pregnant women $(13,14)$, using various technologies, as summarized by $\mathrm{Xu}$ and Eckerman (5). In particular, representing the third generation of anatomic model is the work by Segars, now with Duke University, who used the nonuniform rational b-spline (NURBS) modeling technique (15) that has allowed not only a more realistic rendering of the human body but also the attractive capability of rapid and easy scaling of organs and the whole body. Figure 2 shows the adult male and female NURBS models originally developed by Segars. 
FIGURE 1. Exterior view of one of the Cristy/Eckerman 1987 phantoms (1).

This work describes the development of a complete male and female adult and pediatric (15-y-old, 10-y-old, 5-y-old, 1 -y-old, and newborn) and pregnant woman phantom series for the purposes of internal radiation dosimetry in nuclear medicine using a realistic, NURBS-based body modeling technique, based on the recommended organ, body, and fetal masses in publication 89 of the International Commission on Radiological Protection (ICRP) (16). We have calculated photon- and electron-specific absorbed fractions (SAFs) and dose factors for more than 1,000 radionuclides for this phantom series, as defined in the unified internal and external dose assessment methodology of the RAdiation Dose Assessment Resource (RADAR) for nuclear medicine patient and occupational radiation worker dosimetry (17):

$$
D=\frac{k \tilde{A} \sum_{i} n_{i} E_{i} \phi_{i}}{m}=k \tilde{A} \sum_{i} n_{i} E_{i} \Phi_{i}
$$

where $D$ is absorbed dose in a target organ ( $\operatorname{rad}$ or Gy), $\tilde{\mathrm{A}}$ is cumulated activity (sum of all nuclear transitions that occurred) in a source organ ( $\mu \mathrm{Ci}-\mathrm{h}$ or MBq-s), $n$ is number of radiations with energy $E$ emitted per nuclear transition, $E$ is energy per radiation $(\mathrm{MeV}), \varphi$ is absorbed fraction (fraction of radiation energy absorbed in the target), $m$ is mass of target region ( $\mathrm{g}$ or $\mathrm{kg}), \Phi$ is $\mathrm{SAF}\left(=\varphi / \mathrm{m}\left[\mathrm{g}^{-1}\right.\right.$ or kg $\left.\left.{ }^{-1}\right]\right)$, and $k$ is a proportionality constant ( $\mathrm{rad}-\mathrm{g} / \mu \mathrm{Ci}-\mathrm{h}-\mathrm{MeV}$ or Gy-kg/MBq-s-MeV).

In certain cases (e.g., hollow organs and bone or marrow), results from other models are used to provide a complete dosimetric modeling environment that has been implemented for internal dose assessment in an update of the OLINDA/EXM personal computer code (4).

\section{MATERIALS AND METHODS}

\section{Adult and Pediatric Models}

NURBS models may be easily deformed to represent different sizes and shapes. We developed a series of models representing
FIGURE 2. Anterior views of Segars (15) NURBS models of adult male $(A)$ and adult female (B).

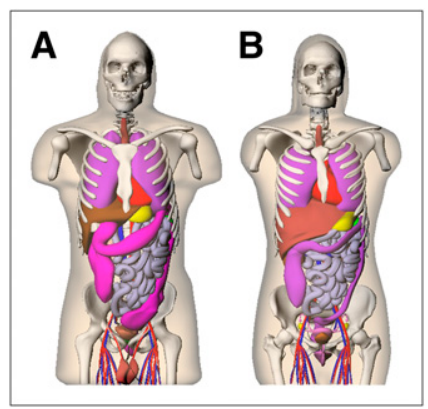

adults and children of different ages scaled from these original adult models to match the recommended age-dependent organ masses given in ICRP publication 89 (16). Existing software tools developed by Segars allow for the rapid scaling of human NURBS. One or more selected organs may be translated or rotated in any direction, scaled linearly in any direction (uniformly in 3 dimensions from the center by a fixed factor), and otherwise modified by the user. Slice-by-slice viewing of results is also afforded by this software tool. We started with Segars' 2 adult models, scaled them to the ICRP 89 reference adult organ masses, and then continued to modify them to represent the smaller models by deforming the surfaces and then adjusting the organs to match the ICRP 89 models for each age. In general, the whole models were not simply scaled equally in all 3 dimensions; for example, the head size is much larger relative to the size of the whole body in young children than in adults. Overall scaling of the body was done to provide an appropriate body size and shape, and then organs were individually scaled. The ICRP 89-recommended organ mass values for adults and children of various reference ages (male and female newborn, 1-y-old, 5-y-old, 10-y-old, 15-y-old, and adult) were used as target masses for individual organs in each phantom. Mostly, organs were varied in all 3 dimensions equally, and then individual organs could be translated if needed to maintain internal consistency between the models. Age-dependent differences (e.g., head size in young children) were incorporated. When finalized, the modified models were rendered in voxel format with another software tool (using a resolution of $1.5 \times 1.5 \times 5 \mathrm{~mm}$ ) and converted from binary to ASCII format. Hollow organs had both wall and content components; target masses from ICRP 89 were applied to both the wall and the content components. Organs were touching and even overlapping in some cases; Segars' models have a priority scheme to handle this, which is invoked during voxelization. All original file sizes were $512 \times 512 \times 512$; these were trimmed to a variable cubic format that eliminated excess rows and columns of voxels outside the phantom and were used with a GEANT4 (GEometry ANd Tracking)-based (18) Monte Carlo radiation transport program that we developed. Separate male and female models were made for the adult and pediatric models. Because the ICRP does not offer separate organ mass values for 10-y-old models and younger, the same model was used for both sexes, with the sex organs added as appropriate. For most organs, the difference between the NURBS reported and voxelized volumes were usually within $1 \%$; for some small organs, however, the differences were sometimes greater. Absorbed fractions were converted to SAFs using the reference organ masses (Tables 1 and 2). Generally, 200,000 starting particles were used at each energy for each source organ. Uncertainties in calculated SAF values were generally less than $5 \%$ for most organs; for some small or distant organs or very 
low-energy particles, the uncertainties were necessarily higher. Reciprocity (19), averaging, and curve-smoothing methods were used to deal with noisier data. Many values at low energies were of high uncertainty or no hits were observed; these were reported as zeroes, and no attempts were made to extrapolate curves beyond the range of the reported data. For the pregnant female models, as noted by Shi et al. (14): "The Monte Carlo run employed approximately $1 \times 10^{7}$ histories to keep the statistical uncertainty to less than $10 \%$ for most of the target organs, except for photon energies below $30 \mathrm{keV}$ and for some target organs that were too small or too far from the source organ. In the latter cases, uncertainties of $10 \%$ or more were observed. The results were discarded if the relative uncertainty was higher than $40 \%$ so we could include as many data points as possible. The cutoff energy for both electron and photon calculations in the EGS4-VLSI user code were set to be 10 keV."

\section{Pregnant Female Models}

A complete description of the methods used to develop SAFs for the pregnant female models developed at Rensselaer Polytechnic Institute (RPI) in collaboration with Vanderbilt has been published previously $(13,14)$. Briefly, organ models were created by extracting the voxel information contained in the so-called boundary representations that consisted of polygonal meshes or NURBS. Individual organs and fetal models were integrated into the adult female whole body by careful adjustment of the organ shape and location to accommodate gestation-related anatomic changes in the body and to avoid organ overlap. For each organ, the volume and mass were specified manually according to reference values recommended in ICRP 89 (16). Once all the organs and total body weights had been adjusted, the surface models were voxelized for dose calculations using the EGS4 Monte Carlo code (20).

\section{RESULTS}

Figure 3 shows images of selected models from the adult and pediatric ICRP 89 phantom series, and Figure 4 shows renderings of the RPI pregnant female models. Figure 5 shows sample photon and electron SAFs for adult and pediatric models. Traditionally, all AFs for electron selfirradiation have been assumed to be 1.0 for an organ irradiating itself and 0.0 for other organs. Although this trend is generally true, when electron transport is performed there is some departure from this assumption at higher energies. This trend is more important for the smaller organs, particularly in the smaller phantoms.

\section{DISCUSSION}

The first generation of stylized anthropomorphic models has served the dosimetry community well for over $40 \mathrm{y}$ for

TABLE 1

Selected Reference Organ Masses (g) for Pediatric/Adult Phantom Series

\begin{tabular}{|c|c|c|c|c|c|c|c|c|c|c|c|c|}
\hline \multirow[b]{2}{*}{ Organ/Tissue } & \multicolumn{2}{|c|}{ Newborn } & \multicolumn{2}{|c|}{$1-y$-old } & \multicolumn{2}{|c|}{ 5-y-old } & \multicolumn{2}{|c|}{$10-y$-old } & \multicolumn{2}{|c|}{ 15-y-old } & \multicolumn{2}{|c|}{ Adult } \\
\hline & Female & Male & Female & Male & Female & Male & Female & Male & Female & Male & Female & Male \\
\hline Adrenals & 6 & 6 & 4 & 4 & 5 & 5 & 7 & 7 & 9 & 10 & 13 & 14 \\
\hline Brain & 380 & 380 & 950 & 950 & 1,180 & 1,310 & 1,220 & 1,400 & 1,300 & 1,420 & 1,300 & 1,450 \\
\hline Breasts & 0 & 0 & 0 & 0 & 0 & 0 & 0 & 0 & 250 & 15 & 500 & 25 \\
\hline Esophagus & 2 & 2 & 5 & 5 & 10 & 10 & 18 & 18 & 30 & 30 & 35 & 40 \\
\hline Eyes & 6 & 6 & 7 & 7 & 11 & 11 & 12 & 12 & 13 & 13 & 15 & 15 \\
\hline Gallbladder wall & 0.5 & 0.5 & 1.4 & 1.4 & 2.6 & 2.6 & 4.4 & 4.4 & 7.3 & 7.7 & 8 & 10 \\
\hline Left colon & 7 & 7 & 20 & 20 & 49 & 49 & 85 & 85 & 122 & 122 & 145 & 150 \\
\hline Small intestine & 30 & 30 & 85 & 85 & 220 & 220 & 370 & 370 & 520 & 520 & 600 & 650 \\
\hline Stomach wall & 7 & 7 & 20 & 20 & 50 & 50 & 85 & 85 & 120 & 120 & 140 & 150 \\
\hline Right colon & 7 & 7 & 20 & 20 & 49 & 49 & 85 & 85 & 122 & 122 & 145 & 150 \\
\hline Heart wall & 20 & 20 & 50 & 50 & 85 & 85 & 140 & 140 & 220 & 230 & 250 & 330 \\
\hline Kidneys & 25 & 25 & 70 & 70 & 110 & 110 & 180 & 180 & 240 & 250 & 275 & 310 \\
\hline Liver & 130 & 130 & 330 & 330 & 570 & 570 & 830 & 830 & 1,300 & 1,300 & 1,400 & 1,800 \\
\hline Lungs & 60 & 60 & 150 & 150 & 300 & 300 & 500 & 500 & 750 & 900 & 950 & 1,200 \\
\hline Muscle & 800 & 800 & 1,900 & 1,900 & 5,600 & 5,600 & 11,000 & 11,000 & 17,000 & 24,000 & 17,500 & 29,000 \\
\hline Ovaries & 0.3 & 0 & 0.8 & 0 & 2 & 0 & 3.5 & 0 & 6 & 0 & 11 & 0 \\
\hline Pancreas & 6 & 6 & 20 & 20 & 35 & 35 & 60 & 60 & 100 & 110 & 120 & 140 \\
\hline Prostate & 0 & 0.8 & 0 & 1 & 0 & 1.2 & 0 & 1.6 & 0 & 4.3 & 0 & 17 \\
\hline Rectum & 3 & 3 & 10 & 10 & 22 & 22 & 40 & 40 & 56 & 56 & 70 & 70 \\
\hline Salivary glands & 6 & 6 & 24 & 24 & 34 & 34 & 44 & 44 & 65 & 68 & 70 & 85 \\
\hline Red marrow & 50 & 50 & 150 & 150 & 340 & 340 & 630 & 630 & 1,000 & 1,080 & 900 & 1,170 \\
\hline Bone surfaces & 4.21 & 4.21 & 13.7 & 13.7 & 33 & 33 & 56 & 56 & 92 & 92 & 120 & 120 \\
\hline Spleen & 9.5 & 9.5 & 29 & 29 & 50 & 50 & 80 & 80 & 130 & 130 & 130 & 150 \\
\hline Testes & 0 & 0.85 & 0 & 1.5 & 0 & 1.7 & 0 & 2 & 0 & 16 & 0 & 35 \\
\hline Thymus & 13 & 13 & 30 & 30 & 30 & 30 & 35 & 40 & 30 & 35 & 20 & 25 \\
\hline Thyroid & 1.3 & 1.3 & 1.8 & 1.8 & 3.4 & 3.4 & 7.9 & 7.9 & 12 & 12 & 17 & 20 \\
\hline $\begin{array}{l}\text { Urinary bladder } \\
\text { wall }\end{array}$ & 4 & 4 & 9 & 9 & 16 & 16 & 25 & 25 & 35 & 40 & 40 & 50 \\
\hline Uterus & 4 & 0 & 1.5 & 0 & 3 & 0 & 4 & 0 & 30 & 0 & 80 & 0 \\
\hline Total body & 3,500 & 3,500 & 10,000 & 10,000 & 19,000 & 19,000 & 32,000 & 32,000 & 53,000 & 56,000 & 60,000 & 73,000 \\
\hline
\end{tabular}


TABLE 2

Selected Reference Organ Masses (g) for RPI Pregnant Female Phantom Series

\begin{tabular}{|lrrr}
\hline \multicolumn{1}{c}{ Organ/Tissue } & 3 mo & 6 mo & 9 mo \\
\hline Adrenals & 13 & 13 & 13 \\
\hline Brain & 1,299 & 1,299 & 1,299 \\
\hline Breasts & 570 & 797 & 906 \\
\hline Fetus & 85 & 1,115 & 3,495 \\
\hline Gallbladder contents & 48 & 48 & 48 \\
\hline Heart contents & 370 & 370 & 370 \\
\hline Heart wall & 250 & 250 & 250 \\
\hline Kidneys & 275 & 275 & 275 \\
\hline Liver & 1,400 & 1,400 & 1,400 \\
\hline Lower intestine contents & 320 & 320 & 320 \\
\hline Lungs & 950 & 950 & 950 \\
\hline Ovaries & 11 & 11 & 11 \\
\hline Pancreas & 120 & 120 & 120 \\
\hline Placenta & 48 & 319 & 650 \\
\hline Small intestine contents & 880 & 880 & 880 \\
\hline Spleen & 130 & 130 & 130 \\
\hline Stomach contents & 231 & 231 & 231 \\
\hline Thymus & 20 & 20 & 20 \\
\hline Thyroid & 17 & 17 & 17 \\
\hline Urinary bladder contents & 129 & 129 & 129 \\
\hline Uterus & 270 & 550 & 1,047 \\
\hline
\end{tabular}

*"Lower intestine" in these models is ascending, transverse, descending, and sigmoid colon, with wall and content sections.

internal and external dose applications (5). Original body models used in dosimetry were spheric (21); this was mathematically convenient and provided conservative estimates of SAFs and doses but was not at all realistic, and there was no provision for organ cross-irradiation. The development of the stylized adult and pediatric phantom series by Cristy and Eckerman (1) (following the original design of Snyder et al. (22)) represented an important incremental change in methods for development of SAFs and a significant increase in model realism over a simple, uniform sphere. Monte Carlo methods were developed to estimate SAF values for the organs, and the science of dose calculations for internal and external simulated sources of radiation was greatly improved. However, the advent of image-based technologies that allowed the development of realistic body models for use in dose calculations brought an exciting opportunity to perform patient-individualized dose calculations in nuclear medicine therapy (23), as well as general dose assessment for internal or external sources. The models presented here represent the third generation of body models, with the organ masses based on those recommended by the ICRP for standardized individuals of various ages (16).

Realistic, voxel-based models derived from real human images have notable differences in organ proximity, compared with the traditional, stylized models, as was illustrated by Stabin and Yoriyaz (24). Organs are closer together, and many are in direct contact with each other (kidneys/adrenals, lungs/heart), whereas in stylized models, separation of organ spaces occurs because of the simplicity of the shapes used to model them. Changes in actual SAF values from the Cristy/ Eckerman model series can be observed, but they are mostly small. This provides indirect validation of these results (because validation using physical phantoms is not possible with available technology); we also checked that the SAFs at low photon energies were approximately 1 /(organ mass). Because the SAFs are similar to those of the previous generation of phantoms, the impact on calculated dose estimates for radiopharmaceuticals, other internal sources, or external sources should be minor but of interest for evaluation. Photon SAFs for lungs $\leftarrow$ liver for all 6 ages in the male phantoms demonstrate trends similar to those of the previous stylized phantoms, but the SAFs for the realistic phantoms are generally a bit higher because of the more realistic modeling of organ proximity (one lung actually rests on top of the liver). At very low energies, the differences are more pronounced, but these photons are typically not important to a complete dose assessment for a given radiopharmaceutical. In the adult female for lungs $\leftarrow$ liver, significant differences are noted at low energies, but at moderate to high energies the values are similar but still slightly higher. For stomach $\leftarrow$ spleen, the NURBS values are several orders of magnitude higher in the $\mathrm{keV}$ range and noticeably higher at all energies. In calculation of dose estimates for individual radiopharmaceuticals, doses to most organs are similar, but doses to
FIGURE 3. Rendering of selected NURBS ICRP 89 phantoms: newborn female model (A), 5-y-old male model (B), 10-y-old female model (C), and 15-y-old male model (D).

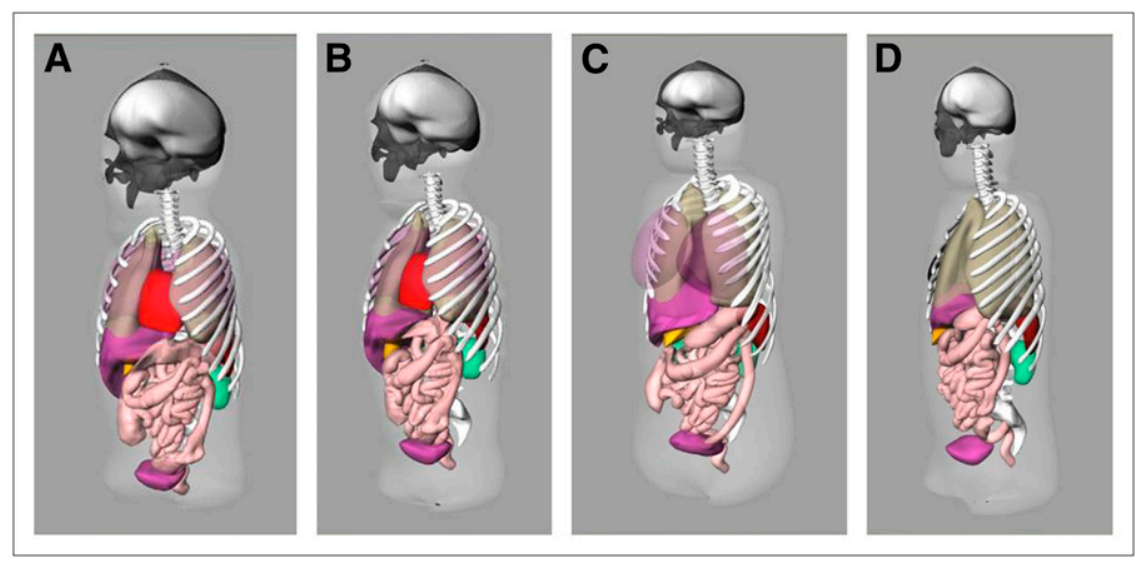




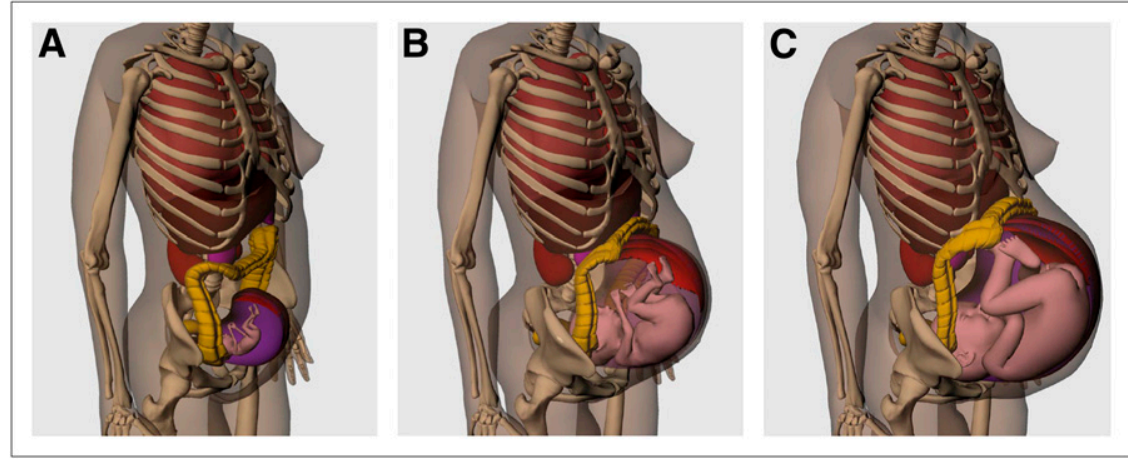

FIGURE 4. Rendering of 3-dimensional organ and body surfaces for RPI pregnant female models: 3 mo (A), 6 mo (B), and 9 mo (C). certain organs (e.g., adrenals and pancreas) may be notably different. However, overall effects on effective dose values are small (to be shown in a subsequent publication).

It is useful to now explicitly treat electron SAFs. The OLINDA/EXM computer code (4) included an approximate correction for loss of electron energy in small objects at high electron energies, based on the AFs developed in unitdensity spheres by Stabin and Konijnenberg (25). Explicit Monte Carlo transport provides a better estimation of this electron energy loss at high energies, which can be noted even for large organs such as kidneys (Fig. 5C) but more dramatically for small organs such as ovaries and testes. Deposition of electron energy on other organs is interesting. In most cases, this is simply a small contribution due to bremsstrahlung radiation, which is known to be an insignificant contribution to the overall dose in any internal dose problem (26). However, in cases in which organs are touching (e.g., kidneys/adrenals), $\beta$-energy is deposited in the first few millimeters of the tissue in the adjacent organ as well (Fig. 5D). Because these SAFs are for whole organs, inclusion of this component is not equivalent to averaging photon energy over the entire organ, which will be generally more uniform. But there is energy deposited, and we decided to leave this contribution in the reported SAFs.

Many other groups, including Petoussi-Henss et al. (6), Kramer et al. (28), Xu et al. (7), Zubal et al. (29), Lee et al. (30), and others, have developed standardized phantoms ultimately adopted by the ICRP (27). The ICRP 110 models also are based on the ICRP 89 organ weights but use manually segmented and adjusted organ representations from 2 human subjects. As such, the numeric values of SAFs from
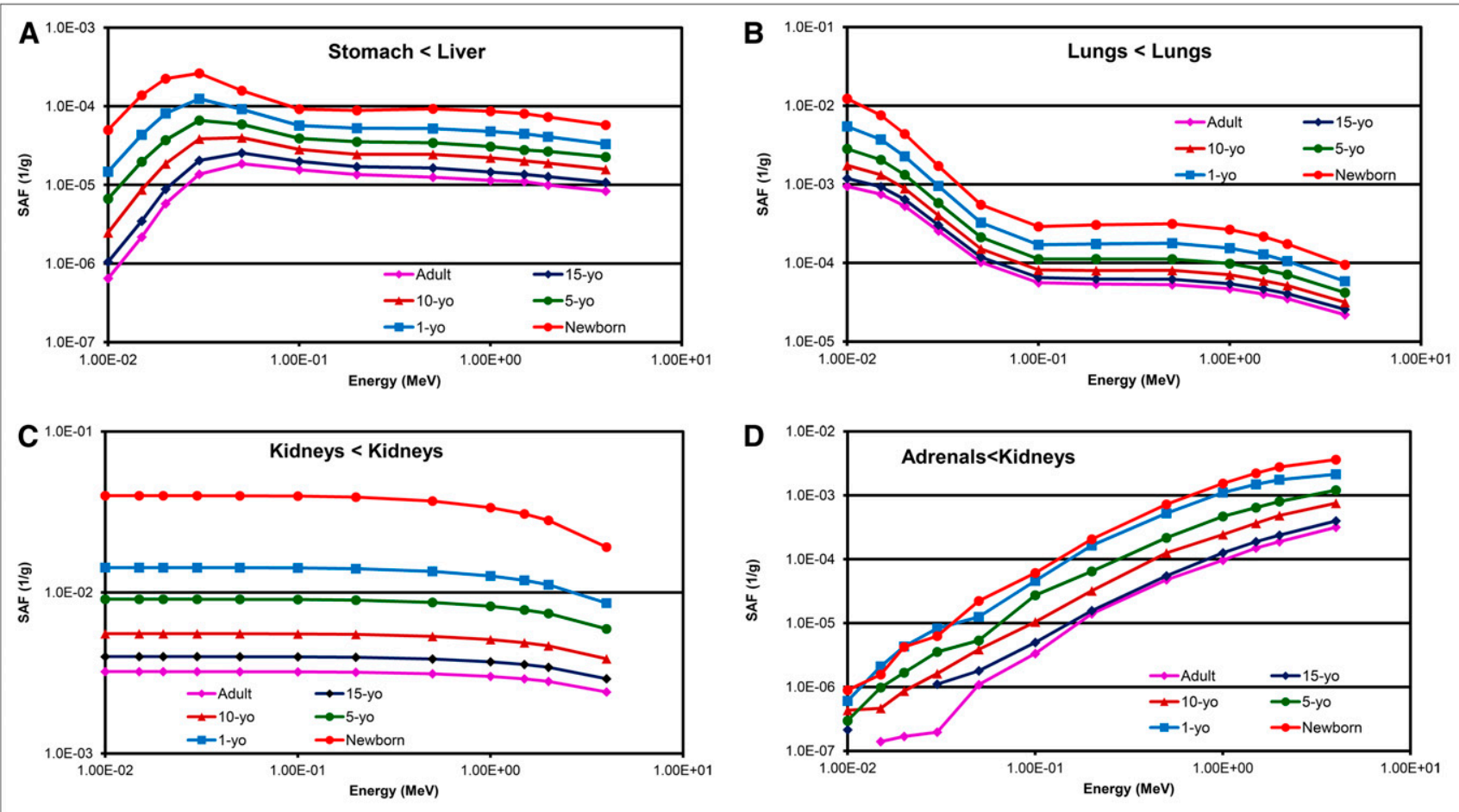

FIGURE 5. Sample SAF plots for ICRP 89 pediatric and adult models: male, photons stomach $\leftarrow$ liver (A), female, photons lungs $\leftarrow$ lungs (B), male, electrons kidneys $\leftarrow$ kidneys $(C)$, and female, adrenals $\leftarrow$ kidneys (D). 
these models should be similar to those in the RADAR phantoms, because both model realistic organ geometries and use the same organ masses. Evaluation of several cases shows that this is the case (details to be provided in a later publication). We maintained the OLINDA/EXM 1.0 and 1.1 bone and marrow model (based on the modified Eckerman model) (31); we feel that this is an advantage in that it provides continuity between the codes in this area. We are aware that better models are under development (32), but they are not complete for all ages and sexes at present. We also maintained the traditional model for electron irradiation of hollow organ walls. The ICRP addressed the issue of the conservatism of assuming that the dose to the wallcontent interface is one half that to the contents, but only for adults (33). We did explicitly model electron and photon transport using hollow-organ contents as the source, but we could not model the exact locations of sensitive cells in the walls, thickness of mucus layers, and other factors. The photon SAFs from the transport were used, but OLINDA/EXM will use the traditional approach for electrons until more complete data are available.

The RADAR phantom series, including the adult, pediatric, and pregnant female models, will be implemented in future versions of the OLINDA/EXM computer code (4), which has facilitated and standardized dose estimates for many years, building on the technical basis of the previous MIRDOSE software (3). New tables of dose estimates for radiopharmaceuticals in current use - organ doses and effective doses-will be generated with these new SAFs and will serve as an update to these dose estimates using this new generation of anthropomorphic phantoms. OLINDA/EXM still treats only average, whole-organ dose, based on an assumed uniform organ uptake. This approach is certainly appropriate for diagnostic applications, but for new drug applications and clinical evaluations this kind of dosimetry is routinely reported for therapeutic radiopharmaceuticals as well. More advanced methods have been demonstrated by several groups as research applications using 3-dimensional distributions of activity to produce nonhomogeneous dose distributions and dose-volume histograms for patientindividualized anatomic models $(23,34)$. There is no present intention for OLINDA/EXM to treat this kind of detailed dosimetry; the role of this code is to provide average organ doses for standardized models.

\section{CONCLUSION}

A new generation of reference models for standardized internal and external dose calculations has been defined. The models will be implemented in standardized software for internal dose calculations and be used to produce new standardized dose estimates for radiopharmaceuticals and other applications.

\section{DISCLOSURE STATEMENT}

The costs of publication of this article were defrayed in part by the payment of page charges. Therefore, and solely to indicate this fact, this article is hereby marked "advertisement" in accordance with 18 USC section 1734.

\section{ACKNOWLEDGMENT}

Work on this project was supported by grant NIH/NCI 1R42CA115122. No other potential conflict of interest relevant to this article was reported.

\section{REFERENCES}

1. Cristy M, Eckerman K. Specific Absorbed Fractions of Energy at Various Ages from Internal Photon Sources. Oak Ridge, TN: Oak Ridge National Laboratory; 1987:1-110. ORNL/TM-8381 V1-V7.

2. Stabin M, Watson E, Cristy M, et al. Mathematical Models and Specific Absorbed Fractions of Photon Energy in the Nonpregnant Adult Female and at the End of Each Trimester of Pregnancy. Oak Ridge, TN: Oak Ridge National Laboratory; 1995:1-140. ORNL/TM-12907.

3. Stabin M. MIRDOSE: the personal computer software for use in internal dose assessment in nuclear medicine. J Nucl Med. 1996;37:538-546.

4. Stabin MG, Sparks RB, Crowe E. OLINDA/EXM: the second-generation personal computer software for internal dose assessment in nuclear medicine. $\mathrm{J} \mathrm{Nucl}$ Med. 2005;46:1023-1027.

5. Xu XG, Eckerman KF. Handbook of Anatomical Models for Radiation Dosimetry. Boca Raton, FL: Taylor and Francis; 2009:1-721.

6. Petoussi-Henss N, Zankl M, Fill U, Regulla D. The GSF family of voxel phantoms. Phys Med Biol. 2002;47:89-106.

7. Xu XG, Chao TC, Bozkurt A. VIP-Man: an image-based whole-body adult male model constructed from color photographs of the Visible Human Project for multi-particle Monte Carlo calculations. Health Phys. 2000;78:476486.

8. Chao TC, Xu XG. Specific absorbed fractions from the image-based VIP-Man body model and EGS4-VLSI Monte Carlo code: internal electron emitters. Phys Med Biol. 2001;46:901-927.

9. Hurtado JL, Lee C, Lodwick D, Goede T, Williams JL, Bolch WE. Hybrid computational phantoms representing the reference adult male and adult female: construction and applications for retrospective dosimetry. Health Phys. 2012; 102:292-304.

10. Kramer R, Zankl M, Williams G, Drexler G. The Calculation of Dose from External Photon Exposures Using Reference Human Phantoms and Monte Carlo Methods. Part I: the Male (ADAM) and Female (EVA) Adult Mathematical Phantoms. Munich, Germany: Gesellschaft für Strahlenund Umweltforschung; 1982. GSF-Bericht-S-885.

11. Schlattl H, Zankl M, Petoussi-Henss N. Organ dose conversion coefficients for voxel models of the reference male and female from idealized photon exposures. Phys Med Biol. 2007;52:2123-2145.

12. Lee C, Lodwick D, Hasenauer D, Williams JL, Lee C, Bolch WE. Hybrid computational phantoms of the male and female newborn patient: NURBS-based whole-body models. Phys Med Biol. 2007;52:3309-3333.

13. Xu XG, Taranenko V, Zhang J, Shi C. A boundary-representation method for designing whole-body radiation dosimetry models: pregnant females at the ends of three gestational periods: RPI-P3, -P6 and -P9. Phys Med Biol. 2007; 52:7023-7044.

14. Shi CY, Xu XG, Stabin MG. SAF values for internal photon emitters calculated for the RPI-P pregnant-female models using Monte Carlo methods. Med Phys. 2008;35:3215-3224.

15. Segars JP. Development and Application of the New Dynamic NURBS-Based Cardiac-Torso (NCAT) Phantom [dissertation]. University of North Carolina, 2001.

16. International Commission on Radiological Protection. ICRP Publication 89: Basic Anatomical and Physiological Data for Use in Radiological Protection: Reference Values. New York, NY: Elsevier Health. 2003:1-265.

17. Stabin MG, Siegel JA. Physical models and dose factors for use in internal dose assessment. Health Phys. 2003;85:294-310.

18. Agostinelli S, Allison J, Amako K, et al. GEANT4: a simulation toolkit. $\mathrm{Nucl}$ Instrum Methods Phys Res A. 2003;506:250-303.

19. Loevinger R, Budinger TF, Watson EE. MIRD primer for absorbed dose calculations. New York, NY: Society of Nuclear Medicine; 1991:1-128.

20. Bielajew AF, Rogers DWO. PRESTA: the parameter reduced electron-step transport algorithm for electron Monte Carlo transport. Nucl Instrum Methods Phys Res B. 1986;18:165-171,174-181. 
21. International Commission on Radiological Protection. Report of Committee II on Permissible Dose for Internal Radiation: ICRP Publication 2. New York, NY: Pergamon Press; 1959:1-233.

22. Snyder W, Ford M, Warner G. Estimates of Specific Absorbed Fractions for Photon Sources Uniformly Distributed in Various Organs of a Heterogeneous Phantom: MIRD Pamphlet No. 5, Revised. New York, NY: Society of Nuclear Medicine; 1978:1-52.

23. Guy MJ, Flux GD, Papavasileiou P, Flower MA, Ott RJ. RMDP: a dedicated package for I-131 SPECT quantification, registration and patient-specific dosimetry. Cancer Biother Radiopharm. 2003;18:61-69.

24. Stabin MG, Yoriyaz H. Photon specific absorbed fractions calculated in the trunk of an adult male voxel-based phantom. Health Phys. 2002;82:21-44.

25. Stabin MG, Konijnenberg M. Re-evaluation of absorbed fractions for photons and electrons in small spheres. $J$ Nucl Med. 2000;41:149-160.

26. Stabin MG, Eckerman KF, Ryman JC, Williams LE. Bremsstrahlung radiation dose in yttrium-90 therapy applications. J Nucl Med. 1994;35:1377-1380.

27. Adult Reference Computational Phantoms: ICRP Publication 110-Annals of the ICRP Volume 39 Issue 2. Maryland Heights, MO: Elsevier; 2009:1-165.

28. Kramer R, Vieira JW, Khoury HJ, Lima FRA, Fuelle D. All about MAX: a male adult voxel phantom for Monte Carlo calculations in radiation protection dosimetry. Phys Med Biol. 2003;48:1239-1262.
29. Zubal IG, Harrell CR, Smith EO, Smith AL, Krischlunas P. High resolution, MRIbased, segmented, computerized head phantom. In: S-Stelson AT, Stabin MG, Sparks RB, eds. Proceedings of the Sixth International Radiopharmaceutical Dosimetry Symposium. Oak Ridge, TN: ORAU; 1996:319-364.

30. Lee C, Lodwick D, Williams JL, Bolch WE. Hybrid computational phantoms of the 15-year male and female adolescent: applications to CT organ dosimetry for patients of variable morphometry. Med Phys. 2008;35:23662382.

31. Stabin MG, Eckerman KF, Bolch WE, Bouchet LG, Patton PW. Evolution and status of bone and marrow dose models. Cancer Biother Radiopharm. 2002;17: $427-433$.

32. Hunt JG, Watchman CJ, Bolch WE. Calculation of absorbed fractions to human skeletal tissues due to alpha particles using Monte Carlo and 3-D chord-based transport techniques. Radiat Prot Dosim. 2007;127:223-226.

33. International Commission on Radiological Protection. Human Alimentary Tract Model for Radiological Protection: ICRP Publication 100. Oxford, U.K.: Pergamon Press; 2007:1-327.

34. Sgouros G, Squeri S, Ballangrud ÅM, et al. Patient-specific, 3-dimensional dosimetry in non-Hodgkin's lymphoma patients treated with ${ }^{131} \mathrm{I}$-anti-B 1 antibody: assessment of tumor dose-response. J Nucl Med. 2003;44:260268. 